Indonesian Journal of Biotechnology, December, 2013

Vol. 18, No. 2, pp.101-115

\title{
Comparative Analysis of Genes Induced by Respiratory Syncytial Virus and DsRNA in Human Epithelial Cells
}

\author{
Gino Valentino Limmon ${ }^{*}$ \\ Faculty of Fisheries and Marine Science, Universitas Pattimura, Ambon-Indonesia
}

\begin{abstract}
Epithelial cells are the primary target of respiratory viral infections and play a pivotal role in virusinduced lung inflammation and in anti viral immune response. A common signal for the presence of viral infections and induction of inflammation is recognition of double stranded RNA (dsRNA). Thus far, there has not been a high-throughput transcrptome analysis of RSV- or dsRNA-induced genes in primary human bronchial epithelial cells (PHBE), nor there has been a comparison between dsRNA- and RSV-induced genes. To establish the transcriptome profiles and to determine the contribution of dsRNA in the induction of inflammation during respiratory virus infection, we compared the gene expression profiles of PHBE cells that were infected with Respiratory Syncytial Virus (RSV) or were treated with dsRNA. Our transcriptome analysis showed that RSV infection and and dsRNA treatment induced up-regulation of 2024 and 159 genes in PHBE respectively. Comparison of genes revealed that RSV and dsRNA commonly induced 75 genes in PHBE cells. The common up-regulated genes were functionally grouped in multiple response pathways involved in inflammation and immune responses. Interestingly, there were several previously unreported genes that were up-regulated in primary human epithelial cells that are relevant to a $\mathrm{TH} 2$ allergic phenotype. This comparison of a high-throughput gene expression study offers a comprehensive view of transcriptional changes induced by dsRNA and RSV, and importantly compares dsRNA-induced genes with RSV-induced genes in PHBE cells.
\end{abstract}

Keywords: RSV, dsRNA, transcriptome, immune response, inflammation

\section{Introduction}

RSV is the most common cause of childhood respiratory viral infections, and can lead to severe bronchiolitis, which is associated with an increase in the induction of asthma (Singh et al., 2007). Previous transcriptomic studies have reported gene expression analysis of RSV-infected cell lines (Zhang et al., 2001), RSV-infected mice (Janssen et al., 2007) and RSV-infected infant whole and cord blood (Fjaerli et al., 2006; Fjaerli et al., 2007). Thus far, there has not been a transciptome analysis of genes that

\section{"Corresponding author:}

Gino Valentino Limmon

Universitas Pattimura, Jl. Ir. M. Putuhena, Kampus Poka-Unpatti, Ambon-Indonesia, Postal code: 97116, Tel: +62911-322691, Fax: +62911-322691 are induced during RSV infection in primary human bronchial epithelial cells, which are the primary target of RSV and other respiratory viruses.

During viral infections, including infections with respiratory viruses, dsRNA can be formed, which acts as a potent inducer of inflammation. Experimentally, dsRNA, both intracellular and extracellular, has been widely used to mimic viral-induced inflammation. Previous high-throughput gene analysis using dsRNA in the form of poly I:C have reported the transcriptomeic changes in human glioma GRE cells (Geiss, 2001), peripheral blood mononuclear cells (PBMC's) (Huang et al., 2006) and rat pancreatic beta cells (Rasschaert et al., 2003). However, to date there has been no characterization or comparison of RSV-and 
dsRNA- induced genes in primary human airway epithelial cells.

In this study, the comprehensive gene expression profiles of PHBE treated with dsRNA or infected with RSV were analyzed using total cellular RNA, hybridized onto high-density oligonucleotide arrays (Agilant Human Array 1A version 2), which contain 20,000 probes. Interestingly, ours results reveal novel RSV- and dsRNA-induced genes. Some of the novel RSV-induced genes identified in our result including ADAM8 and IL13RA2. ADAM8 has been reported to be induced in experimental asthma in mice, and in patients with severe and moderate asthma (Foley et al., 2007; King et al., 2004; Matsuno et al., 2006; Richens et al., 2007) and IL-13RA2 is important for IL-13 signaling and TGF- $\mu 1-$ mediated lung fibrosis (FichtnerFeigl et al., 2007; Fichtner-Feigl et al., 2006).

We also identified novel dsRNA induced genes such as SOCS3 and PRDM1 (BLIMP-1). A report by Seki et al. (2003) showed that SOCS3 was important in the induction and maintenance of allergic $\mathrm{TH} 2$ reactions. BLIMP-1 is required for epidermal cell c-myc regulation and for the development of the effector function of B and T lymphocytes ( Lin, 2000; Lin, 1997; Martins, 2008; Messika et al., 1998; Zhou et al., 2007).

Importantly, study showed that 75 genes were commonly induced by RSV infection or dsRNA treatment in PHBE cells. These genes were functionally grouped in multiple response pathways such as inflammation, apoptosis, transcription regulation, cell signaling, and cell differentiation. The induction of gene expression was confirmed by determination of changes in expression of select proteins.

This is, to our knowledge, is the first study that has analyzed global gene expression in PHBE cells after dsRNA treatment and RSV infection. This characterization of the common genes validates the use of dsRNA as viral mimetic to detect a subset of proinflammatory signal during RSV infection, and may likely contribute to developing specific anti-inflammatory agents.
The aim of this study is to unravel the gene expression profile of human epithelial cell induced by RSV infection and DsRNA treatment.

\section{Materials and Methods Cells, tissue culture conditions, antibodies and reagents.}

The primary human bronchial epithelial (PHBE) were grown as a monolayer at $37^{\circ} \mathrm{C}$ in a $5 \% \mathrm{CO} 2$ humidified chamber in a serumfree epithelial cell growth medium from Cambrex (Lonza, Walkersville, MD). The synthetic dsRNA, poly I:C, was purchased from Sigma Chemicals (St. Louis, MO), dissolved in phosphate-buffered saline, and was used at concentrations indicated in each figure legend. The dsRNA structural fidelity of poly I: $C$ was determined by the ability of each dsRNA batch to activate the dsRNAspecific protein kinase PKR in in vitro kinase assays (Imani, 1988).

\section{RNA extraction and RT-PCR}

For RNA extraction, cells were left untreated, were treated with $1 \mu \mathrm{g} / \mathrm{ml}$ poly I:C, or were infected with RSV at multiplicity of infection(MOI) of 2.5 plaqueforming unit (pfu)/ cell. After $2 \mathrm{hr}$ for dsRNA and $24 \mathrm{hr}$ for RSV, total cellular RNA was isolated using the TRIzol reagent (Invitrogen, Carlsbad, CA) according to the manufacturers' instructions. The quality of RNA was evaluated by electrophoresis and only high quality RNA was used in the microarray and RT-PCR experiments.

For real-time PCR, first strand cDNA was synthesized using superscript reverse transcriptase (Invitrogen, Carlsbad, CA) using $1 \mu \mathrm{g}$ total RNA. Following reverse transcription, $1 \mu \mathrm{l}$ of cDNA was amplified by real-time PCR. Each experiment was performed in duplicate in 96 well plates by using 1x Sybr Green master mix (BioRad, Hercules, CA) in a final volume of 25 $\mu 1$. Amplifications were performed with the following protocol: $95^{\circ} \mathrm{C}$ for 3 minutes followed by 50 cycles of $94^{\circ} \mathrm{C}$ for $10 \mathrm{sec}$ and $60^{\circ} \mathrm{C}$ for $30 \mathrm{sec}$. 
Microarray, linear amplification, labeling Protocol and feature extraction

Gene expression analysis was conducted using Agilent Human1Av2 arrays (Agilent Technologies, Palo Alto, CA). Total RNA was amplified using the Agilent Low RNA Input Fluorescent Linear Amplification Kit protocol. Starting with 500ng of total RNA, Cy3 or Cy5 labeled cRNA was produced according to manufacturer's protocol. For each two color comparison, $750 \mathrm{ng}$ of each Cy3 and Cy5 labeled cRNAs were mixed and fragmented using the Agilent In Situ Hybridization Kit protocol. Hybridizations were performed for 17 hours in a rotating hybridization oven using the Agilent 60-mer oligo microarray processing protocol. Slides were washed as indicated in this protocol and then scanned with an Agilent Scanner. Data was obtained using the Agilent Feature Extraction software (v7.5), using defaults for all parameters.

\section{Rosetta Resolver, Genespring GX, and Ingenuity pathways analysis.}

Images and GEML files, including error and p-values, were exported from the Agilent Feature Extraction software and deposited into Rosetta Resolver (version 4.0, build 4.0.1.0.7. RSPLIT) (Rosetta Biosoftware, Kirkland, WA). The resultant ratio profiles were combined into ratio experiments as described (Dai et al., 2002). Intensity plots were generated for each ratio experiment and genes were considered "signature genes" if the $p$ value was $<0.001$.

Data from Agilent Feature Extraction software were also loaded to genespring (version 7.3.1) and filtered for markers and flagged spots. The data were then adjusted for dye swap experiment, normalized and filtered based on fold change.

Genes that differentially regulated by dsRNA and RSV infection in primary epithelial cell, BEAS-2B and A549 were analyzed through the use of Ingenuity Pathways Analysis (Ingenuity Systems, www.ingenuity.com).
The Functional Analysis identified the biological functions and/or diseases that were most significant to the data set. Genes from the dataset that met the normalized cut off of 1.5 fold and were associated with biological functions and/or diseases in the Ingenuity Pathways Knowledge Base were considered for the analysis. Fischer's exact test was used to calculate a p-value determining the probability that each biological function and/or disease assigned to that data set is due to chance alone.

The Functional Analysis of a network identified the biological functions and/or diseases that were most significant to the genes in the network. The network genes associated with biological functions and/ or diseases in the Ingenuity Pathways Knowledge Base were considered for the analysis. Fischer's exact test was used to calculate a p-value determining the probability that each biological function and/ or disease assigned to that network is due to chance alone.

\section{Cytokine and chemokine protein determination.}

The amount of cytokines and chemokines present in media and cell extract was determined using a multiplexed cytokines assay system (Bio-plex 200) (Biorad, Hercules, CA). In brief, premixed antibody-coupled beads were reconstituted in assay buffer and loaded onto a pre-wetted 96 well plate. The beads were washed once with the provided wash buffer and then diluted standard or samples were added, mixed by vortexing and incubated at room temperature for $60 \mathrm{~min}$. After washing 3 times to remove unbound proteins, a second specific biotinylated detection antibody was added to the beads, mixed and incubated for an additional $30 \mathrm{~min}$. For final detection, after washing, streptavidinphycoerythrin (streptavidin-PE) was added to the mixture and incubated for $10 \mathrm{~min}$. The beads were then washed, resuspended in assay buffer and analyzed using Bio- 
Table 1. List of PCR primers used to validate microarray data

\begin{tabular}{|c|c|c|}
\hline \multirow[t]{2}{*}{1} & BBC3-FWD & tgtgaccactggcattcatt \\
\hline & BBC3-REV & tcctccetcttccgagattt \\
\hline \multirow[t]{2}{*}{2} & BF-FWD & atgctgacccaatacttgc \\
\hline & BF-REV & tccactactcccagctgat \\
\hline \multirow[t]{3}{*}{3} & BIRC3FWD & tgagcccaggagtttgaatc \\
\hline & BIRC3REV & acaccatgtccctaaaatgtca \\
\hline & & GTGTGCGCAAATCCAAA \\
\hline \multirow[t]{3}{*}{4} & CCL20-FWD & $\mathrm{AC}$ \\
\hline & & CAAGTCCAGTGAGGCAC \\
\hline & CCL20-REV & AAA \\
\hline \multirow[t]{2}{*}{5} & CSF2-FWD & cttcctgtgcaacccagatt \\
\hline & CSF2-REV & ccatcctgagtttctagctcttg \\
\hline \multirow[t]{2}{*}{6} & CXCL-1-FWD & cccttggacattttatgtctttc \\
\hline & CXCL-1-REV & aacatcaataattaagccctttg \\
\hline \multirow[t]{2}{*}{7} & CXCL-10-FWD & cctctcccatcacttccetac \\
\hline & CXCL-10-REV & gctgatttggtgaccatcatt \\
\hline \multirow[t]{2}{*}{8} & CXCL-11-FWD & gggtgaaaggaccaaaaaca \\
\hline & CXCL-11-REV & acaacaagtaagaacgtgaaagca \\
\hline \multirow[t]{2}{*}{9} & CXCL2-FWD & gaggctgaggaatccaagaa \\
\hline & CXCL2-REV & tgttgaccacacactgtgaaa \\
\hline \multirow[t]{3}{*}{10} & CXCL-3-FWD & caacatttttatgctgaagtttcc \\
\hline & CXCL-3-REV & ttccaagggaaagagaaacg \\
\hline & & AGT GCT GAA AAC AAA \\
\hline \multirow[t]{3}{*}{11} & DUSP1-FWD & CCT GCT \\
\hline & & CCA GTA TTA TTC CCG \\
\hline & DUSP-1-REV & ACG ACA \\
\hline \multirow[t]{3}{*}{12} & EFNA1-FWD & gggatggagaaagaagtgga \\
\hline & EFNA1-REV & cagctcccactgtgcctta \\
\hline & & TGTTTGGCTTATAAACA \\
\hline \multirow[t]{3}{*}{13} & EGR1-FWD & CATTGAA \\
\hline & & CCCAATCGCAGCTACTTT \\
\hline & EGR1-REV & ATTT \\
\hline \multirow[t]{2}{*}{14} & G1P2-FWD & caccgtgttcatgaatctgc \\
\hline & G1P2-REV & ctttatttccggeccttgat \\
\hline \multirow[t]{2}{*}{15} & IFIT1-FWD & cctccttgggttcgtctaca \\
\hline & IFIT1-REV & ggctgatatctgggtgccta \\
\hline \multirow[t]{2}{*}{16} & IFIT2-FWD & tgcttggaacgattgagattt \\
\hline & IFIT2-REV & caggcaaacattttccaacc \\
\hline \multirow[t]{3}{*}{17} & IFIT4-FWD & gtggttgtgacgggtaggag \\
\hline & IFIT4-REV & ctcccaaagtgctgggatta \\
\hline & & CAG CAG TTC CAG AAG \\
\hline \multirow[t]{3}{*}{18} & IFNB-FWD & GAG GA \\
\hline & & AGC CAG TGC TCG ATG \\
\hline & IFNB-REV & AAT CT \\
\hline \multirow[t]{3}{*}{19} & IL1A-FWD & caccctatattttgcatagtaccaa \\
\hline & IL1A-REV & tcctaggtctgtggcttcttg \\
\hline & & ATT CTG CGC AGC TTT \\
\hline \multirow[t]{3}{*}{20} & IL-6-FWD & AAG GA \\
\hline & & ATC TGA GGT GCC CAT \\
\hline & IL-6-REV & GCT AC \\
\hline
\end{tabular}

\begin{tabular}{|c|c|c|}
\hline \multirow[t]{2}{*}{21} & IL8-FWD & tctggcaacctagtctgct \\
\hline & IL8-REV & gcttccacatgtcctcacaa \\
\hline \multirow[t]{2}{*}{22} & INHBA-FWD & accaagctgagacccatgtc \\
\hline & INHBA-REV & ttgccactgtcttctctgga \\
\hline \multirow[t]{2}{*}{23} & NFKBIA-FWD & ttcatggaaggaccacatttt \\
\hline & NFKBIA-REV & caccatttacaggagggtaaca \\
\hline \multirow[t]{2}{*}{24} & NFKBIZ-FWD & ctaagtctttgtagataaagcagatgg \\
\hline & NFKBIA-REV & ttcaatgcatgagaaaaagca \\
\hline \multirow[t]{2}{*}{25} & RIG-I-FWD & tagtttgcggtttcctggac \\
\hline & RIG-I-REV & cactgctcaccagattgcat \\
\hline \multirow[t]{3}{*}{26} & TNFAIP3-FWD & taccctggtattgggacagc \\
\hline & TNFAIP3-REV & acagcaaccacaaagcacac \\
\hline & & CAC TGC CCC TCC AGT \\
\hline \multirow[t]{3}{*}{27} & ZFP36-FWD & TTT TA \\
\hline & ZFP36-REV & $\begin{array}{l}\text { TGA TTT ATG GGT GGG } \\
\text { GTT TG }\end{array}$ \\
\hline & ADAM8-FWD & $\begin{array}{l}\text { GCAACCAC AAGCAGGA } \\
\text { GTG }\end{array}$ \\
\hline 28 & ADAM8-REV & $\begin{array}{l}\text { CAGA ACCACCACCACGA } \\
\text { AG } \\
\text { TTGCGTAAGCCAAACAC }\end{array}$ \\
\hline \multirow[t]{3}{*}{29} & IL13RA2-FWD & $\begin{array}{l}\text { CTA } \\
\text { CTALAACCAAACAC }\end{array}$ \\
\hline & & TGAACATTTGGCCATGA \\
\hline & IL13RA2-REV & CTG \\
\hline \multirow[t]{2}{*}{30} & RND3- FWD & $\begin{array}{l}\text { AATAGAGTTGAGCCTGT } \\
\text { GGG }\end{array}$ \\
\hline & RND3-REV & $\begin{array}{l}\text { CTAATGTACTAACATCT } \\
\text { GTCCGC }\end{array}$ \\
\hline \multirow[t]{2}{*}{31} & BMP2-FWD & $\begin{array}{l}\text { TGCACCAAGATGAACA } \\
\text { CAG }\end{array}$ \\
\hline & BMP2-REV & GCTGTTTGTGTTTGGCTTG \\
\hline \multirow[t]{2}{*}{32} & CCNA1-FWD & $\begin{array}{l}\text { GTTGTGCTGGCTACAGT } \\
\text { GG }\end{array}$ \\
\hline & CCNA1-REV & $\begin{array}{l}\text { CCTGCTCTAGTTCATCCA } \\
\text { TGTA }\end{array}$ \\
\hline \multirow[t]{2}{*}{33} & ADRA2C-FWD & $\begin{array}{l}\text { GTGGTGATCGCCGTGCTG } \\
\text { AC }\end{array}$ \\
\hline & ADRA2C-REV & $\begin{array}{l}\text { CGTTTTCGGTAGTCGGGG } \\
\text { AC }\end{array}$ \\
\hline
\end{tabular}

Plex 200 system. The concentration of each cytokine was then determined by measuring magnitude of the fluorescently labeled reporter molecules associated with individual target proteins.

\section{Results \\ Identification of RSV-induced genes in PHBE cells \\ To identify Genes that induced by double stranded RNA (Poly I:C), global gene expression profiling were performed}


by using labeled cRNA derived from total RNA extracted from mock-infected or RSVinfected (2.5 pfu/cell) PHBE cells after 24 hr. The cRNA was hybridized onto Agilent Human1Av2 arrays. Each comparison (mock vs infected) was done in duplicate with dye swap experiments. Data were then vigorously filtered to remove the biases. The genes that have more than 1.5 relative fold inductions compare to untreated control were considered differentially regulated genes.

Our result shows that RSV infection induced up-regulation of 2024 genes in PHBE cells. Genes that were highly induced by RSV included, cytokines and chemokines involved in innate and adaptive immunity, matrix metalloproteinases, cell signaling molecules, TGF family members, cell cycle regulatory molecules, and heat shock proteins (see supplement for complete list). We next compared our data with the previously published RSV-induced genes in other cells. The comparison revealed several novel RSVinduced genes that are listed in Table 2. The novel RSV-induced genes that are shown in Table 1 included ADAM8, ADRAC2C, and IL13RA2. This genes are associated with induction of experimental asthma in mice, and are up-regulated in patients with severe and moderate asthma (Foley et al., 2007; King et al., 2004; Matsuno et al., 2006)

\section{Identification of dsRNA induced genes in PHBE cells}

To determine the dsRNA induced gene profiles, PHBE cell were either left untreated or were treated with dsRNA at $1 \mu \mathrm{g} / \mathrm{ml}$ for $2 \mathrm{hr}$ prior to RNA extraction. Gene expression profiling was carried out as above. The results showed that expression of 159 genes in primary epithelial cells was induced by dsRNA treatment (see supplement for complete list). DsRNA induced genes included inflammatory and regulatory cytokines and chemokines, signal transduction molecules, cell migration molecules, and apoptosis pathways. Some of the highly induced gene included interferon

Table 2. List of significant diseases and cellular function with related genes identified by IPA .

\begin{tabular}{|c|c|c|}
\hline Function & $\begin{array}{c}-\log \\
\text { (p-value) }\end{array}$ & Molecules \\
\hline $\begin{array}{l}\text { Inflammatory } \\
\text { Response }\end{array}$ & 9.73E-15-4.59E-06 & $\begin{array}{l}\text { IL1A, PTGER4, CXCL10, PLAU, ICAM1, TNFAIP3, CCL5, MMP13, IL8, } \\
\text { IL23A, CXCL2, ZFP36, NFKBIA, DUSP1, TNF, SOCS3, SOD2, CXCL3, } \\
\text { CSF2, EGR1, IL6 }\end{array}$ \\
\hline Viral Function & 1.95E-10-9.13E-06 & IFIH1, NFKBIA, CXCL10, TNF, CXCL3, CCL5, RIPK2, TNIP1, IL6, IL8 \\
\hline $\begin{array}{l}\text { Immunological } \\
\text { Disease }\end{array}$ & 2.28E-09-6.12E-06 & $\begin{array}{l}\text { IER3, PTGER4, CXCL10, IL7R, ICAM1, CCL5, PRDM1, IL23A, IL8, } \\
\text { EFNA1, NFKBIA, DUSP1, KLF2, TNF, SOCS3, SOD2, BIRC3, CSF2, } \\
\text { BMP2, EGR1, IL6 }\end{array}$ \\
\hline Respiratory & & \\
\hline Disease & 3.34E-07-8.08E-07 & TNF, CXCL3, ICAM1, CSF2, EGR1, IL6 \\
\hline Cell Death & 3.27E-16-1.28E-05 & $\begin{array}{l}\text { IL1A, IER3, PMAIP1, NEDD9, PTGER4, MMP13, SDC4, KLF6, IL8, } \\
\text { ZFP36, EFNA1, NFKBIA, PPP1R15A (includes EG:23645), CXCL3, } \\
\text { RIPK2, BTG1, INHBA, PLAUR, BMP2, EGR1, TNFAIP8, IL6, ATF3, } \\
\text { PLK3, HSPA1A, PLAU, ICAM1, IL7R, TNFAIP3, CCL5, PRDM1, IFIH1, } \\
\text { CXCL1, HBEGF, ELF3, DUSP1, KLF2, SOCS3, TNF, SOD2, BIRC3, CSF2 }\end{array}$ \\
\hline & & $\begin{array}{l}\text { IER3, IL1A, PMAIP1, PTGER4, IFIT3, KLF6, ISG15, IL8, IL28B, EFNA1, } \\
\text { CXCL2, NFKBIA, PPP1R15A (includes EG:23645), CXCL3, RIPK2, } \\
\text { INHBA, PLAUR, BTG1, BMP2, EGR1, TNFAIP8, IL6, ATF3, PLK3, }\end{array}$ \\
\hline $\begin{array}{l}\text { Cellular Growth } \\
\text { and Proliferation }\end{array}$ & 1.27E-15-1.14E-05 & $\begin{array}{l}\text { HSPA1A, CXCL10, PLAU, IL29, ICAM1, IL7R, PIM3, CCL5, PRDM1, } \\
\text { CD83, IL23A, IFIH1, CXCL1, HBEGF, DUSP1, ELF3, KLF2, TNF, SOCS3, } \\
\text { SOD2, CSF2, ISG20 }\end{array}$ \\
\hline Cell Cycle & 1.8E-07-1.21E-05 & $\begin{array}{l}\text { PLK3, IER3, IL1A, NEDD9, PLAU, KLF6, IL8, NFKBIA, HBEGF, DUSP1, } \\
\text { PPP1R15A (includes EG:23645), TNF, SOD2, CSF2, PLAUR, INHBA, } \\
\text { BMP2, EGR1, IL6, ATF3 }\end{array}$ \\
\hline
\end{tabular}


Table 3. Novel RSV induced genes

\begin{tabular}{|c|c|c|c|c|c|}
\hline & Gene ID & $\begin{array}{l}\text { Gene } \\
\text { Name }\end{array}$ & $\begin{array}{c}\text { Fold } \\
\text { Change }\end{array}$ & Description & Location \\
\hline 1 & NM_018661 & DEFB103A & 37.91 & defensin, beta 103A & Extracellular Space \\
\hline 2 & NM_007036 & ESM1 & 14.71 & endothelial cell-specific molecule 1 & Extracellular Space \\
\hline 3 & NM_001458 & FLNC & 13.72 & filamin C, gamma (actin binding protein 280 ) & Cytoplasm \\
\hline 4 & NM_001109 & ADAM8 & 12.09 & ADAM metallopeptidase domain 8 & Plasma Membrane \\
\hline 5 & NM_000683 & ADRA2C & 10.74 & adrenergic, alpha-2C-, receptor & Plasma Membrane \\
\hline 6 & NM_001323 & CST6 & 9.20 & cystatin E/M & Extracellular Space \\
\hline 7 & NM_004633 & IL1R2 & 7.69 & interleukin 1 receptor, type II & Plasma Membrane \\
\hline 8 & NM_025245 & PBX4 & 6.75 & pre-B-cell leukemia homeobox 4 & Nucleus \\
\hline 9 & NM_174873 & $\mathrm{P} 2 \mathrm{R} \times 2$ & 6.71 & purinergic receptor $\mathrm{P} 2 \mathrm{X}$ & Plasma Membrane \\
\hline 10 & ВC008283 & CCK & 6.30 & cholecystokinin & Extracellular Space \\
\hline 11 & NM_004636 & SEMA3B & 6.28 & semaphorin 3B & Extracellular Space \\
\hline 12 & NM_001305 & CLDN4 & 6.14 & claudin 4 & Plasma Membrane \\
\hline 13 & ВС015503 & AGR2 & 5.94 & anterior gradient homolog 2 & Extracellular Space \\
\hline 14 & NM_003033 & ST3GAL1 & 5.80 & ST3 beta-galactoside alpha-2,3-sialyltransferase 1 & Cytoplasm \\
\hline 15 & NM_000952 & PTAFR & 5.72 & platelet-activating factor receptor & Plasma Membrane \\
\hline 16 & NM_015277 & NEDD4L & 5.35 & $\begin{array}{l}\text { neural precursor cell expressed, } \\
\text { developmentally down-regulated } 4\end{array}$ & Cytoplasm \\
\hline 17 & NM_001874 & $\mathrm{CPM}$ & 5.30 & carboxypeptidase $\mathrm{M}$ & Plasma Membrane \\
\hline 18 & NM_016354 & SLCO4A1 & 5.26 & $\begin{array}{l}\text { solute carrier organic anion transporter family, } \\
\text { member } 4 \mathrm{~A} 1\end{array}$ & Plasma Membrane \\
\hline 19 & AK000158 & CA12 & 5.25 & carbonic anhydrase XII & Plasma Membrane \\
\hline 20 & NM_003914 & CCNA1 & 5.23 & cyclin A1 & Nucleus \\
\hline 21 & NM_032957 & RTEL1 & 5.12 & regulator of telomere elongation helicase 1 & Nucleus \\
\hline 22 & NM_006043 & HS3ST2 & 5.07 & $\begin{array}{l}\text { heparan sulfate (glucosamine) 3-O- } \\
\text { sulfotransferase } 2\end{array}$ & Cytoplasm \\
\hline 23 & NM_013259 & TAGLN3 & 5.02 & transgelin 3 & Unknown \\
\hline 24 & NM_000640 & IL13RA2 & 5.01 & interleukin 13 receptor, alpha 2 & Plasma Membrane \\
\hline
\end{tabular}

induced genes (IFIT1, IFIT2, IFIT4), TNF- $\alpha$ induced gene (TNFAIP2), apoptosis inhibitor (BIRC3), eph receptor (EFNA1) and several cytokines and chemokines (IL6, IL-8, CXCL1, CXCL2, CXCL3, PTX3).

We also identified several novel dsRNA induced genes in PHBE cells. The list of novel dsRNA induced genes is presented in Table 3. Some of these genes such as BMP2, GBP, and RND3 have been implicated in inflammation.

BMP2 has been shown to be induced and may play a role in BMP/SMAD signaling during airway inflammation (Rosendahl et al., 2002), and RND3/RhoE was reported to be involved in inflammatory response by regulating IRAK/ERK/NF-KB pathway and cytoskeleton organization (Guasch et al., 2007).

\section{Comparison of differentially expressed genes in dsRNA treated cells and RSV infected cells}

Since dsRNA is commonly used as viral mimetic, we compared the gene expression profiles of RSV-infected and dsRNA-treated PHBE cells. Data from Venn diagram comparing RSV- and dsRNA-induced genes (Figure 1) revealed that 75 genes were induced in common. The genes that were commonly up-regulated were listed in Table 4. Several genes that are involved in innate immune responses that were highly induced by both RSV and dsRNA in PHBE cells included apoptosis gene (BIRC3), chemokines (IL-8, CXCL1, CXCL11), and interferon induced protein (IFIT1, IFIT2, IFIT4, ISG15). This data indicate that dsRNA can mimic an innate 
Table 4. Novel dsRNA induced genes

\begin{tabular}{clllll}
\hline & Gene ID & $\begin{array}{c}\text { Gene } \\
\text { Name }\end{array}$ & $\begin{array}{c}\text { Fold } \\
\text { Change }\end{array}$ & Description & Location \\
\hline 1 & NM_019618 & IL1F9 & 3.03 & interleukin 1 family, member 9 & Extracellular Space \\
2 & NM_018724 & IL20 & 2.02 & interleukin 20 & Extracellular Space \\
3 & NM_052941 & GBP4 & 3.89 & guanylate binding protein 4 & Cytoplasm \\
4 & NM_018284 & GBP3 & 2.93 & guanylate binding protein 3 & Cytoplasm \\
5 & NM_004120 & GBP2 & 2.57 & guanylate binding protein 2, interferon-inducible & Cytoplasm \\
6 & NM_005168 & RND3 & 2.14 & Rho family GTPase 3 & Cytoplasm \\
7 & NM_006018 & GPR109B & 4.99 & G protein-coupled receptor 109B & Plasma Membrane \\
8 & NM_001200 & BMP2 & 2.46 & bone morphogenetic protein 2 & Extracellular Space \\
9 & NM_152439 & BEST3 & 3.76 & bestrophin 3 & Unknown \\
10 & NM_000891 & KCNJ2 & 3.44 & potassium inwardly-rectifying channel J2 & Plasma Membrane \\
11 & NM_173200 & NR4A3 & 2.08 & nuclear receptor subfamily 4A3 & Nucleus \\
12 & NM_004484 & GPC3 & 4.62 & glypican 3 & Plasma Membrane \\
13 & NM_019016 & KRT24 & 3.88 & keratin 24 & Cytoplasm \\
14 & NM_025047 & ARL14 & 3.41 & ADP-ribosylation factor-like 14 & Unknown \\
15 & NM_006813 & PNRC1 & 2.49 & proline-rich nuclear receptor coactivator 1 & Nucleus \\
16 & NM_014350 & TNFAIP8 & 2.45 & tumor necrosis factor, alpha-induced protein 8 & Cytoplasm \\
17 & NM_020652 & ZNF286A & 2.28 & zinc finger protein 286A & Nucleus \\
18 & NM_001198 & PRDM1 & 2.90 & PR domain containing 1 & Nucleus \\
\hline
\end{tabular}

inflammatory subset of genes that are upregulated during RSV infection.

\section{Validation of microarray result with real time PCR}

To validate the results of the gene array experiments, we used RT-PCR and Bio-Plex assay. Initially the novel RSV-induced genes (ADAM8, IL13RA2, CCNA1 and ADRA2C) novel dsRNA-induced genes (RAND3, BMP2

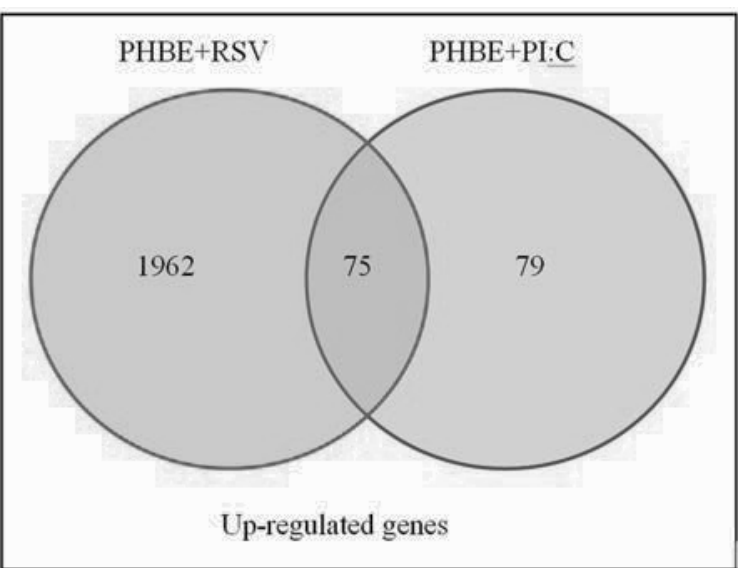

Figure 1. Venn diagram of differentially expressed genes. Comparison of genes induced by RSV infection and dsRNA treatment in PHBE cells. and SOCS3), and several commonly induced genes were verified by RT-PCR (Figure 2). We also verified 28 genes from the 75 genes that were found to be up-regulated by RSV and dsRNA in PHBE cells and all of the 27 genes that were found to be up-regulated by RSV and dsRNA in PHBE cells and all of the 27 genes that were shown to beup-regulated by microarray analysis were proven to be upregulated by Sybr Green RT-PCR (Table 5).

We next confirmed up-regulation of several cytokines and chemokines by protein determination using Bio-Plex assay, this

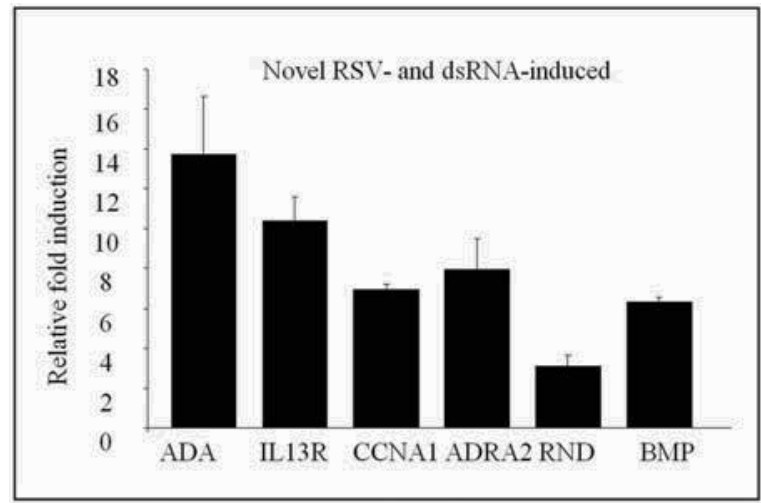

Figure 2. RT-PCR validation of novel RSV- and dsRNAinduced genes. 
Table 5. Genes that were commonly induced by DsRNA and RSV in PHBE.

\begin{tabular}{|c|c|c|c|c|c|c|}
\hline & Gene ID & Gene Name & PHBE+PI:C & t-test $p$-value & PHBE+RSV & t-test $p$-value \\
\hline 1 & NM_001547 & IFIT2 & 57.11 & 0.00914 & 11.01 & $6.12 \mathrm{E}-05$ \\
\hline 2 & NM_001548 & IFIT1 & 21.33 & 0.0133 & 11.21 & 8.72E-06 \\
\hline 3 & NM_000584 & IL8 & 15.47 & 0.00791 & 12.52 & 7.49E-05 \\
\hline 4 & NM_005409 & CXCL11 & 13.22 & 0.00879 & 12.83 & $6.99 \mathrm{E}-05$ \\
\hline 5 & NM_000594 & TNF & 11.73 & 0.000855 & 4.491 & 0.000318 \\
\hline 6 & NM_006291 & TNFAIP2 & 11.36 & 0.055 & 4.016 & 0.00237 \\
\hline 7 & NM_001565 & CXCL10 & 11.32 & 0.0179 & 3.228 & 0.00515 \\
\hline 8 & NM_002090 & CXCL3 & 10.61 & 0.0252 & 4.565 & 0.00157 \\
\hline 9 & NM_006290 & TNFAIP3 & 10.51 & 0.0417 & 2.476 & 0.0145 \\
\hline 10 & NM_001549 & IFIT4 & 10.05 & 0.00175 & 4.968 & 0.00245 \\
\hline 11 & ВС032663 & PMAIP1 & 8.979 & 0.0161 & 3.882 & 5.13E-21 \\
\hline 12 & NM_000600 & IL6 & 8.817 & 0.0232 & 1.911 & 0.0445 \\
\hline 13 & NM_001511 & CXCL1 & 8.533 & 0.0139 & 6.731 & 4.37E-05 \\
\hline 14 & NM_020529 & NFKBIA & 7.578 & 0.0217 & 3.455 & 0.00402 \\
\hline 15 & NM_138397 & LINCR & 7.363 & 0.0378 & 1.966 & 0.0392 \\
\hline 16 & NM_001511 & CXCL1 & 5.994 & 0.0169 & 3.951 & 0.00253 \\
\hline 17 & NM_002089 & CXCL2 & 5.693 & 0.0786 & 2.471 & 0.0146 \\
\hline 18 & NM_005101 & G1P2 & 5.686 & 0.0273 & 9.094 & 0.000185 \\
\hline 19 & NM_001165 & BIRC3 & 5.581 & 0.0613 & 28.13 & $1.59 \mathrm{E}-07$ \\
\hline 20 & ВС009507 & ISG15 & 5.525 & 0.000307 & 7.542 & 0.000322 \\
\hline 21 & ВС006322 & ATF3 & 5.231 & 0.000144 & 3.306 & 0.00473 \\
\hline 22 & NM_004428 & EFNA1 & 5.136 & 0.0208 & 2.228 & 0.0224 \\
\hline 23 & NM_006018 & GPR109B & 4.987 & 0.0471 & 11.96 & 0.000135 \\
\hline 24 & NM_000758 & CSF2 & 4.964 & 0.00017 & 11.11 & 0.0392 \\
\hline 25 & NM_002192 & INHBA & 4.622 & 0.0117 & 8.515 & 0.000224 \\
\hline 26 & NM_172139 & IL28B & 4.577 & 0.0205 & 2.067 & 0.0229 \\
\hline 27 & NM_052941 & GBP4 & 3.887 & 0.22 & 2.004 & 0.0488 \\
\hline 28 & NM_003733 & OASL & 3.695 & 0.000456 & 7.352 & 0.00035 \\
\hline 29 & NM_004417 & DUSP1 & 3.522 & 0.00383 & 3.681 & 0.00321 \\
\hline 30 & NM_004428 & EFNA1 & 3.5 & 0.0642 & 1.686 & 0.0825 \\
\hline 31 & NM_000636 & SOD2 & 3.45 & 0.000578 & 5.162 & 0.00103 \\
\hline 32 & NM_000958 & PTGER4 & 3.337 & 0.152 & 1.945 & 0.0416 \\
\hline 33 & NM_000575 & IL1A & 3.146 & 0.000829 & 2.027 & 0.0343 \\
\hline 34 & NM_006417 & IFI44 & 2.967 & 0.0522 & 9.982 & 0.000142 \\
\hline 35 & AF038963 & RIG-I & 2.959 & 0.004 & 11.36 & 0.00603 \\
\hline 36 & NM_001198 & PRDM1 & 2.896 & 0.00794 & 2.906 & 0.00762 \\
\hline 37 & NM_016584 & IL23A & 2.665 & 0.0845 & 79.9 & 0.0125 \\
\hline 38 & NM_012118 & CCRN4L & 2.53 & 0.126 & 1.703 & 0.0786 \\
\hline 39 & NM_006813 & PNRC1 & 2.486 & 0.016 & 3.293 & 0.00485 \\
\hline 40 & NM_001200 & BMP2 & 2.458 & 0.00221 & 3.743 & 0.00303 \\
\hline 41 & NM_014350 & TNFAIP8 & 2.45 & 0.0312 & 2.104 & 0.029 \\
\hline 42 & NM_000201 & ICAM1 & 2.44 & 0.021 & 15.9 & 0.00286 \\
\hline 43 & NM_003407 & ZFP36 & 2.377 & 0.00455 & 3.605 & 0.00345 \\
\hline 44 & NM_003955 & SOCS3 & 2.263 & 0.0103 & 3.328 & 0.014 \\
\hline 45 & NM_004233 & CD83 & 2.194 & 0.00374 & 1.685 & 0.0828 \\
\hline 46 & NM_001945 & DTR & 2.133 & 0.00142 & 3.551 & 0.00365 \\
\hline
\end{tabular}




\begin{tabular}{lllllll}
\hline 47 & NM_016270 & KLF2 & 2.066 & 0.00492 & 1.872 & 0.0493 \\
48 & NM_172138 & IL28A & 2.061 & 0.108 & 1.817 & 0.0568 \\
49 & NM_002999 & SDC4 & 2.005 & 0.349 & 2.051 & 0.0293 \\
50 & NM_002659 & PLAUR & 1.985 & 0.128 & 5.033 & 0.00338 \\
51 & NM_014330 & PPP1R15A & 1.973 & 0.187 & 2.139 & 0.0266 \\
52 & NM_004556 & NFKBIE & 1.968 & 0.0296 & 4.053 & 0.0023 \\
53 & BC017083 & PIM3 & 1.891 & 0.00494 & 2.462 & 0.0148 \\
54 & AK056293 & IFIH1 & 1.88 & 0.1 & 4.157 & 0.00211 \\
55 & NM_020651 & PELI1 & 1.837 & 0.00837 & 1.674 & 0.0854 \\
56 & NM_001964 & EGR1 & 1.77 & 0.045 & 1.793 & 0.0608 \\
57 & NM_001731 & BTG1 & 1.761 & 0.0419 & 3.277 & 0.00486 \\
58 & NM_006403 & NEDD9 & 1.735 & 0.0177 & 1.82 & 0.0575 \\
59 & NM_001300 & COPEB & 1.733 & 0.011 & 1.998 & 0.0362 \\
60 & A_23_P57836 & A_23_P57836 & 1.71 & 0.0122 & 2.27 & 0.0206 \\
61 & NM_153341 & IBRDC3 & 1.701 & 0.247 & 2.564 & 0.00519 \\
62 & NM_006403 & NEDD9 & 1.662 & 0.212 & 2.989 & 0.00694 \\
63 & NM_004433 & ELF3 & 1.656 & 0.102 & 2.674 & 0.0105 \\
64 & NM_004073 & PLK3 & 1.655 & 0.0168 & 3.122 & 0.00582 \\
65 & AB014515 & N4BP1 & 1.651 & 0.0432 & 1.625 & 0.0996 \\
66 & NM_002985 & CCL5 & 1.648 & 0.0382 & 1.98 & 0.0379 \\
67 & NM_002185 & IL7R & 1.648 & 0.0155 & 2.14 & 0.0269 \\
68 & NM_006058 & TNIP1 & 1.634 & 0.0121 & 3.308 & 0.00471 \\
69 & NM_031866 & FZD8 & 1.621 & 0.307 & 2.645 & 0.0111 \\
70 & NM_003897 & IER3 & 1.614 & 0.00857 & 2.826 & 0.00847 \\
71 & NM_130781 & RAB24 & 1.595 & 0.0196 & 2.43 & 0.0156 \\
72 & NM_172140 & IL29 & 1.584 & 0.0205 & 2.269 & 0.0206 \\
73 & NM_002658 & PLAU & 1.582 & 0.02 & 6.92 & 0.00042 \\
74 & NM_002201 & ISG20 & 1.579 & 0.0109 & 9.915 & 0.0196 \\
75 & AF099011 & EHD1 & 1.577 & 0.0109 & 3.771 & 0.00296 \\
\hline
\end{tabular}

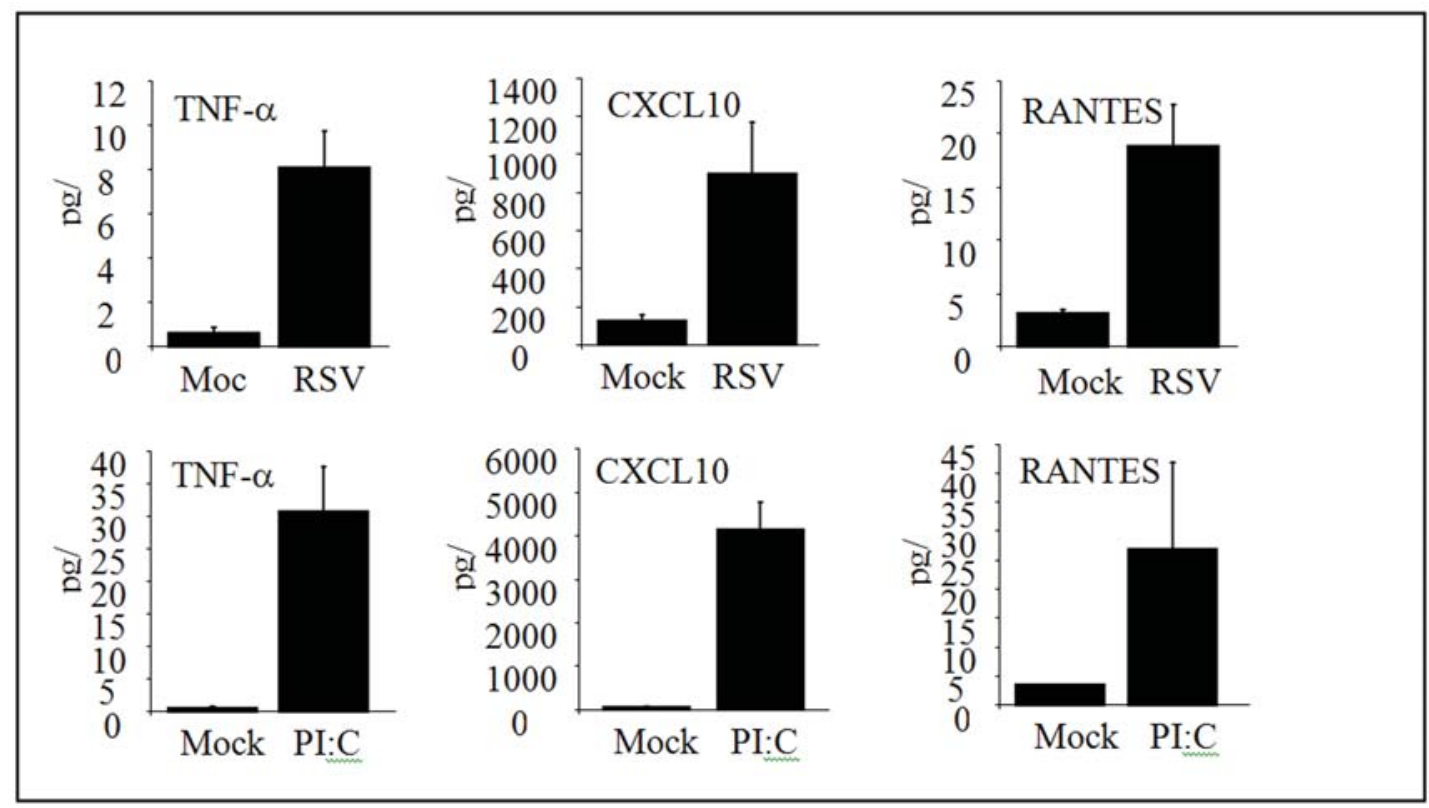

Figure 3. Analysis of protein expression. 


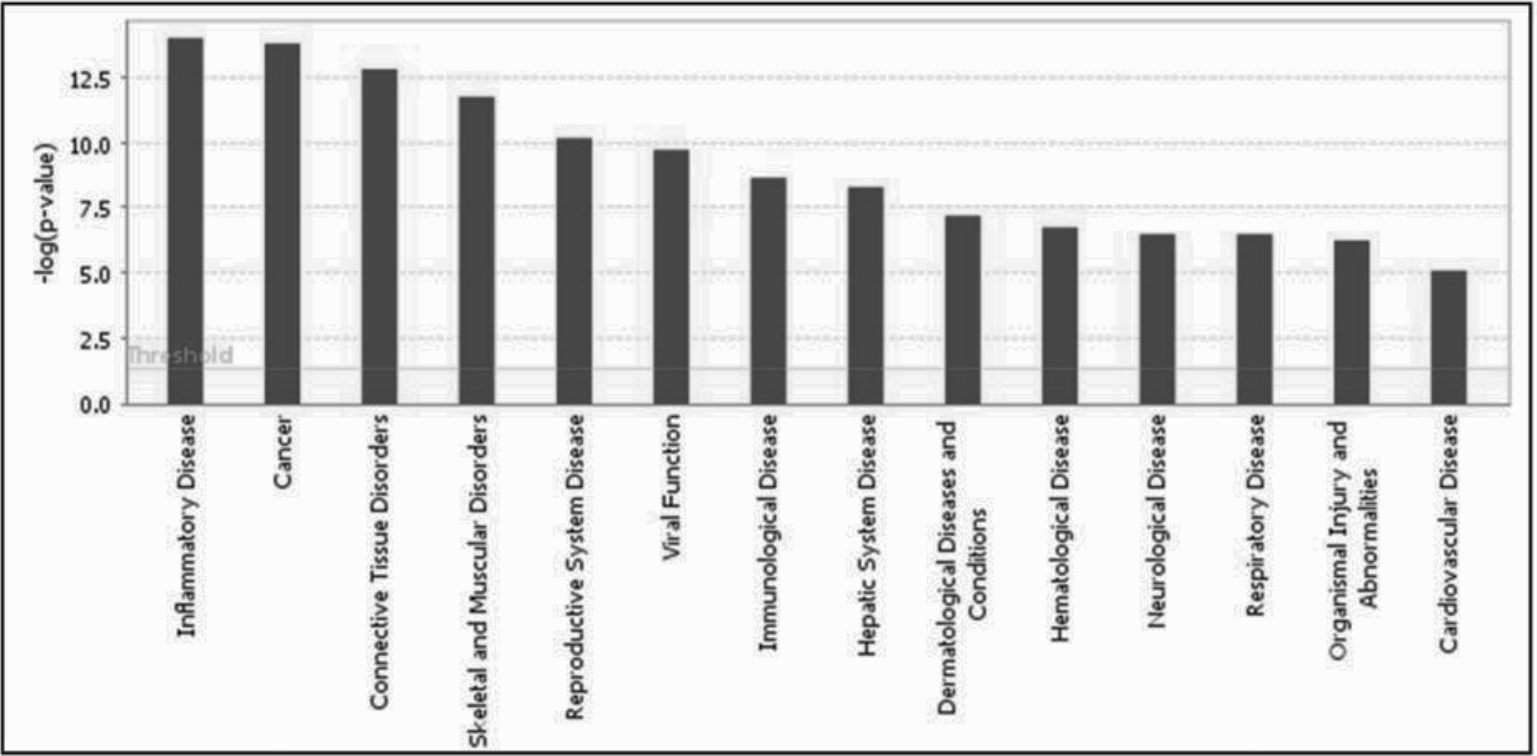

Figure 4A. Functional analysis of differentially expressed genes.

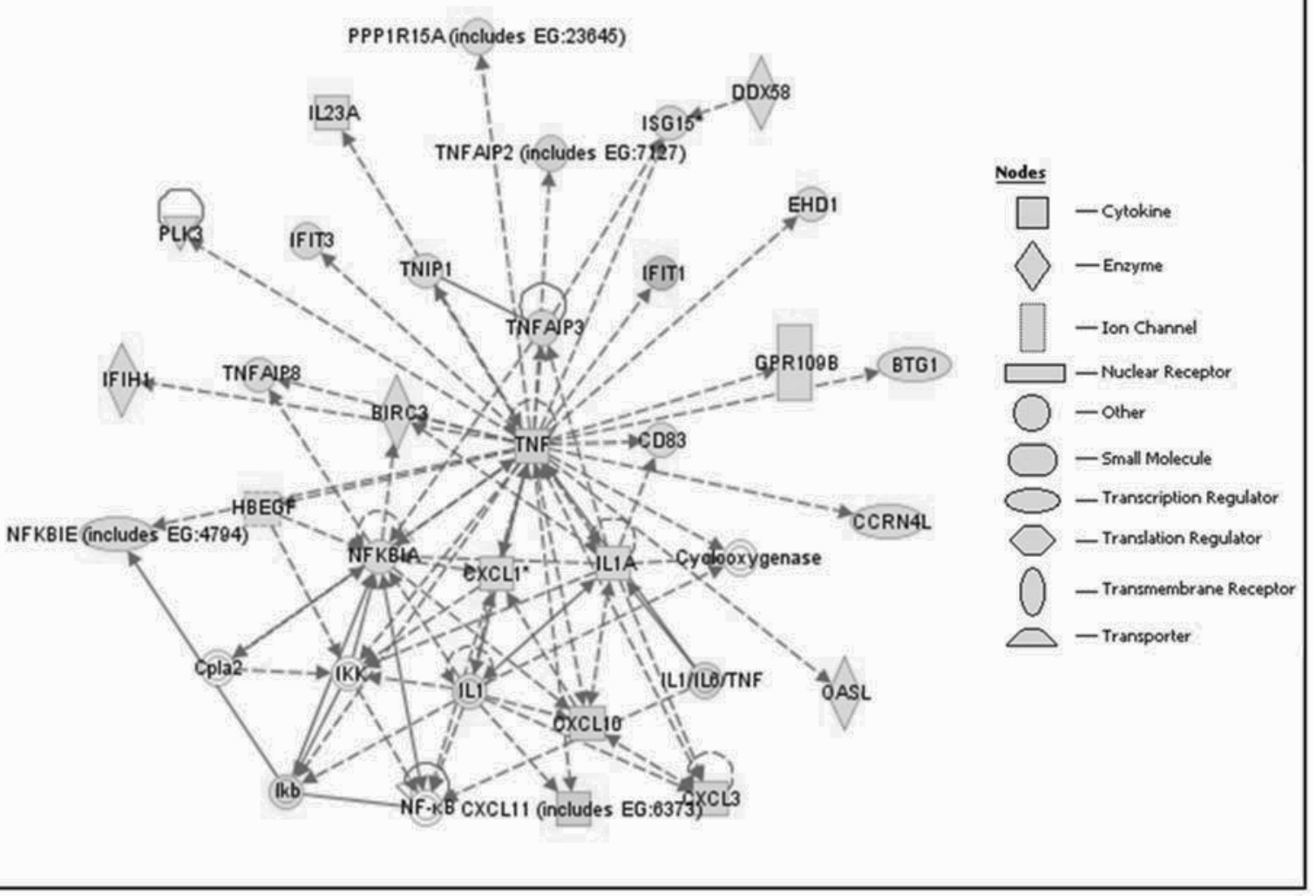

Figure 4B. Functional network analysis of the genes induced by dsRNA and RSV using IPA. Functional network of genes that was commonly induced by RSV infection and dsRNA treatment. The top-scoring network consists of nodes, where each node represents a gene. The shape of the node is indicative of the functional class of the gene product. Multiple connections indicate the biological relationships between the nodes. 
included TNF- $\alpha$, IP10/CXCL10 and RANTES/ CCL5. Data in Figure 3 showed that cytokine protein expression was significantly increased in infected or treated cells, which validated the data obtained from microarray experiments.

\section{Functional analysis of differentially expressed genes}

To investigate the relation and interaction of genes/molecules that were differentially regulated by dsRNA and RSV, we analyzed the up-regulated genes with Ingenuity Pathways Analysis (IPA, Ingenuity Systems). Functional analysis of 75 genes that were commonly induced by RSV and dsRNA in PHBE showed that the up-regulated genes are involved in various diseases and cellular activation pathways. The highest significant diseases and molecular pathways corresponded to these genes are inflammatory disease Figure 4A. From 75 commonly upregulated genes, 20 genes were determined by IPA to be related to inflammatory disease (Table 1 ).

TNF- $\alpha$ has been reported to be induced by dsRNA in vitro and in vivo, and is among the most important cytokines that play a role in viral induced inflammation. Therefore, particularly important nodes in the network are the genes that are known to interact or induced by TNF- $\alpha$ (Figure 4B).

\section{Discussion}

During viral infections, a large repertoire of genes is induced to prevent viral replication and spread. The initial site of infection for respiratory viruses is the airway epithelium; therefore, determining the viral-induced transcriptional events in these cells can shed lights on the initial steps leading to anti viral immune response. The establishment of the anti viral state requires coordinate transcriptional activation of many genes of innate and adaptive immunity. Previous reports have shown that viral infection of various cell types or treatment with poly I:C induced several genes, including type I IFN's. TNF- $\alpha$ RANTES, IL-1, IL-4, IL-6 and IL-8.
DsRNA is recognized as signal to the presence of viral infection. Experimentally, dsRNA in the form of poly I:C has been widely used as a viral mimetic to induce an anti-viral state. Thus far, there have been several reports on the transcriptomic profiling of virus infections and dsRNA treatment in cells. What has been lacking is a global expression profiling in PHBE cells, which are the natural target of respiratory viral infections. In addition, there has not been a comparison between RSV infection and dsrNA treatment to assess the contribution of dsRNA-induced events during virus infections in these cells.

Our global transcriptomic analysis identified 2024 genes that were upregulated by RSV and 159 genes that were induced by dsRNA treatment. A comparison of these genes revealed that 75 genes were commonly up-regulated in PHBE cells after treatment with dsRNA or infection with RSV. Interestingly, dsRNA induced similar pattern of inflammatory cytokines and chemokines to RSV infection. These included type I IFN's, TNF- $\alpha$, IL-1, IL-6, IL-8, CCL-5, CXCl1, CXCL2, CXCL3, CXCL10, and CXCL11. However there were a number of genes that were specifically induced only by dsRNA or RSV (see table 3). Our results also revealed several dsRNA- and RSV-induced genes that have not been reported to be induced in in epithelial cells. These novel genes that are induced by RSV and or dsRNA included ADAMs, ILR13R2, CCNA1, ADRA2C, and BMP2, RND3, SOCS3 and BLMP-1.

ADAMs such as ADAM8 and ADAM10 are disintegrins metalloproteinases that play an important role in releasing soluble CD23 membrane bound precursor (Fourie et al., 2003). The release of the soluble form of CD23 has been reported to increase IgE production (Christie et al., 1997; Kilmon et al., 2001; Saxon et al., 1990), which is the key component of allergic reactions. In as much $\operatorname{IgE}$ is critical for allergic reactions, this observation is consistent with previous reports showing that RSV and dsRNA are 
Table 6. RT-PCR validation of gene expression.

\begin{tabular}{|c|c|c|c|c|c|}
\hline & Gene ID & Gene Name & PHBE + PI:C & PHBE + RSV & Family \\
\hline 1 & NM_014417 & BBC3 & 374.81 & 2.26 & Other \\
\hline 2 & NM_001710 & $\mathrm{BF}$ & 6.82 & 1.48 & peptidase \\
\hline 3 & NM_001165 & BIRC3 & 4.10 & 59.51 & Other \\
\hline 4 & NM_004591 & CCL20 & 492.13 & 1.89 & cytokine \\
\hline 5 & NM_000758 & CSF2 & 22.78 & 1518.56 & cytokine \\
\hline 6 & NM_001511 & CXCL1 & 6.17 & 11.66 & cytokine \\
\hline 7 & NM_001565 & CXCL10 & 46.05 & 84.37 & cytokine \\
\hline 8 & NM_005409 & CXCL11 & 5.62 & 47.73 & cytokine \\
\hline 9 & NM_002089 & CXCL2 & 2.32 & 2.82 & cytokine \\
\hline 10 & NM_002090 & CXCL3 & 9.32 & 21.33 & cytokine \\
\hline 11 & NM_004417 & DUSP1 & 8.52 & 5.02 & phosphatase \\
\hline 12 & NM_004428 & EFNA1 & 3.67 & 1.71 & Other \\
\hline 14 & NM_001964 & EGR1 & 5.01 & 1.48 & transcription regulator \\
\hline 15 & NM_005101 & G1P2 & 35.27 & 8.73 & Other \\
\hline 16 & NM_001548 & IFIT1 & 5.05 & 6.99 & Other \\
\hline 17 & NM_001547 & IFIT2 & 12.60 & 6.30 & Other \\
\hline 18 & NM_001549 & IFIT4 & 4.68 & 5.35 & Other \\
\hline 19 & NM_002176 & IFNB1 & 58.00 & 3.76 & cytokine \\
\hline 20 & NM_000575 & IL1A & 2.78 & 2.38 & cytokine \\
\hline 21 & NM_000600 & IL6 & 8.00 & 16.58 & cytokine \\
\hline 22 & NM_000584 & IL8 & 30.59 & 46.88 & cytokine \\
\hline 23 & NM_002192 & INHBA & 3.92 & 17.10 & growth factor \\
\hline 24 & NM_020529 & NFKBIA & 5.74 & 3.61 & transcription regulator \\
\hline 25 & NM_031419 & NFKBIZ & 3.29 & 1.22 & transcription regulator \\
\hline 26 & AF038963 & RIG-I & 1.87 & 4.73 & enzyme \\
\hline 27 & NM_006290 & TNFAIP3 & 12.73 & 4.17 & Other \\
\hline 28 & NM_003407 & ZFP36 & 4.48 & 9.59 & transcription regulator \\
\hline
\end{tabular}

associated with induction of allergic immune responses (Akira, 2006; Graham, 2000; von Herrath, 2003). Based on epidemiologic and experimental evidence, expression of ADAM8 has been shown to be up-regulated in a murine model of asthma, and in patients with severe and moderate asthma (Foley et al., 2007; King et al., 2004; Matsuno et al., 2006). Taken together, these data may suggest a novel mechanism for the association of virus infections with induction and exacerbations of allergic diseases such as asthma.

Another interesting gene that has not been reported previously that was upregulated by RSV in ours transcriptomic analysis was the alpha 2 subunit of IL-13 receptor (IL-13RA2). Recent reports have shown that IL-13RA2 maybe decoy receptor for IL-13 which can abrogate a stimulatory signal generated from IL-13 and and IL13RA1 interaction ( $\mathrm{Wu}, 2003)$. IL-13 is a pleiotrophic cytokine with potent immune and epithelial cell-regulatory functions such as suppression of inflammation, epithelial growth, and mucus secretion (Hershey, 2003; Holgate, 2003; Kasaian, 2008). Based on the accumulated data, it is tempting to speculate that up-regulation of the decoy IL13RA2 during RSV infection maybe underlying factor for the immuno-deviation and severe bronchiolitis associated with this infection. Furthermore, IL-13RA has been suggested to play important role in TGF- $\beta 1$ mediated fibrosis and could be considered for new therapeutic target (Fichtner-Feigl et al., 2007; Fichtner-Feigl et al., 2006). 
A pathway analysis or our data using IPA revealed that TNF- $\alpha$ was a central player in the virus- and dsRNA-induced events (see figure 4B). TNF- $\alpha$ is an early and potent inducer of inflammation, which can regulate innate and adaptive immune responses, monocyte and lymphocyte migration, and tissue damage. We previously reported that induction of TNF- $\alpha$ during RSV infection or dsRNA treatment was regulated by dsRNAactivated protein kinase (PKR) and p28 MAPK activation (Meusel, 2003). In addition, several of the intracellular antiviral pathways are also up-regulated including, RIG-1, MDA5, TLR3 and MX-1. Predictably, our data suggest that a coordinated activation of several anti-viral pathways may be required for an effective response against respiratory viral infections

Collectively, our data showed that dsRNA could mimic several of the inflammatory and regulatory cytokines and chemokines that RSV induces. Our data further provide several novel genes that are induced by dsRNA and RSV, which may be associated with the induction of allergic conditions. Our future studies are aimed at further characterizing the regulation of these novel RSV- and dsRNA induced genes in airways epithelial cells.

The expression of the Novel genes that were up-regulated in primary human lung epithelial cell after RSV infection or dsRNA treatment by microarray analysis was confirmed by RT-PCR. Y axis represents the relative fold increase of expression. $X$ axis represents genes.

The protein expression level of selected genes were analyzed using bioplex assay. Consistent with the increase in RNA expression, protein expression levels of the selected genes also significantly increase.

Shown are selected Ingenuity Pathway Analysis (IPA) assigned functional categories for the genes induced by dsRNA and RSV. The -log (significance) indicates an increase in confidence for each category.

\section{Acknowledgement}

This research was supported by the Intramural Research Program of the NIH/ National Institute of Environmental Health Sciences.

\section{References}

Akira, S., S. Uematsu, and O. Takeuchi., 2006. Pathogen recognition and innate immunity. Cell., 124(4), p. 783-801

Christie, G. et al., 1997. IgE secretion is attenuated by an inhibitor of proteolytic processing of CD23 (Fc epsilonRII). Eur J Immunol., 27(12), p. 3228-35.

Dai, H., et al., 2002. Use of hybridization kinetics for differentiating specific from non-specific binding to oligonucleotide microarrays. Nucleic Acids Res., 30(16), p. e86.

Fichtner-Feigl, S. et al., 2006. IL-13 signaling through the IL-13alpha2 receptor is involved in induction of TGF-beta1 production and fibrosis. Nat Med., 12(1), p. 99-106.

Fichtner-Feigl, S. et al., 2007. Induction of IL13 triggers TGF-beta1-dependent tissue fibrosis in chronic 2,4,6-trinitrobenzene sulfonic acid colitis. J Immunol., 178(9), p. 5859-70.

Fjaerli, H.O et al., 2006. Whole blood gene expression in infants with respiratory syncytial virus bronchiolitis. BMC Infect Dis., 6, p. 175.

Fjaerli, H.O., et al. 2007. Cord blood gene expression in infants hospitalized with respiratory syncytial virus bronchiolitis. J Infect Dis., 196(3), p. 394-404.

Foley, S.C. et al., 2007. Increased expression of ADAM33 and ADAM8 with disease progression in asthma. J Allergy Clin Immunol., 119(4), p. 863-71.

Fourie, A.M. et al., 2003. Catalytic activity of ADAM8, ADAM15, and MDC-L (ADAM28) on synthetic peptide substrates and in ectodomain cleavage of CD23. J Biol Chem., 278(33), p. 30469-77.

Geiss, G.J., G. Guo, J. Bumgarner, R. Katze, M. G. and Sen, G. C., 2001. A Comprehensive 
View of Regulation of Gene Expression by Double-stranded RNA-mediated Cell Signaling. J. Biol. Chem., 267 (32), p. 30178-30182.

Graham, B.S., T.R. Johnson, and R.S. Peebles., 2000. Immune-mediated disease pathogenesis in respiratory syncytial virus infection. Immunopharmacology., 48 (3), p. 237-47.

Guasch, R.M. et al., 2007. RhoE participates in the stimulation of the inflammatory response induced by ethanol in astrocytes. Exp Cell Res., 313(17), p. 3779-88.

Hershey, G.K., 2003. IL-13 receptors and signaling pathways: an evolving web. J Allergy Clin Immunol., 111(4), p. 677-90; quiz 691.

Holgate, S.T. et al., 2003. Mechanisms of airway epithelial damage: epithelialmesenchymal interactions in the pathogenesis of asthma. Eur Respir J Suppl., 44, p. 24s-29s.

Huang, C C. et al., 2006. A pathway analysis of poly(I:C)-induced global gene expression change in human peripheral blood mononuclear cells. Physiol Genomics., 26(2), p. 125-33.

Imani, F. and B.L. Jacobs., 1988. Inhibitory activity for the interferon-induced protein kinase is associated with the reovirus serotype 1 sigma 3 protein. Proc Natl Acad Sci U S A., 85(21), p. 7887-91.

Janssen, R. et al., 2007. Host transcription profiles upon primary respiratory syncytial virus infection. J. Virol., 81(11), p. 5958-67.

Kasaian, M.T. and D.K. Miller., 2008. IL-13 as a therapeutic target for respiratory disease. Biochem Pharmacol.

Kilmon, M.A. et al., 2001. Regulation of IgE production requires oligomerization of CD23. J Immunol., 167(6), p. 3139-45.

King,N.E. etal.,2004. Expressionand regulation of a disintegrin and metalloproteinase (ADAM) 8 in experimental asthma. Am J Respir Cell Mol Biol., 31(3), p. 257-65.

Lin, K.I., Y. Lin, and K. Calame., 2000. Repression of c-myc is necessary but not sufficient for terminal differentiation of B lymphocytes in vitro. Mol Cell Biol., 20(23), p. 8684-95.

Lin, Y., K. Wong, and K. Calame., 1997. Repression of c-myc transcription by Blimp-1, an inducer of terminal B cell differentiation. Sci., 276 (5312), p. 596-9.

Martins, G. and K. Calame., 2008. Regulation and functions of Blimp-1 in T and B lymphocytes. Annu Rev Immunol., 26, p. 133-69.

Matsuno, O. et al., 2006. Role of ADAM8 in experimental asthma. Immunol Lett., 102(1), p. 67-73.

Messika, E.J. et al., 1998. Differential effect of B lymphocyte-induced maturation protein (Blimp-1) expression on cell fate during B cell development. J Exp Med., 188(3), p. 515-25.

Meusel, T.R.a.F.I., 2003. Viral induction of inflammatory cytokines in human epithelial cells follows a p38 mitogenactivated protein kinase-dependent but NF-kappa B-independent pathway. J. Immunol., 171(7), p. 3768-74.

Rasschaert, J. et al., 2003. Global profiling of double stranded RNA- and IFN-gammainduced genes in rat pancreatic beta cells. Diabetologia., 46(12), p. 1641-57.

Richens, J. et al., 2007. The detection of ADAM8 protein on cells of the human immune system and the demonstration of its expression on peripheral blood B cells, dendritic cells and monocyte subsets. Immunobiol., 212(1), p. 29-38.

Rosendahl, A. et al., 2002. Activation of bone morphogenetic protein/Smad signaling in bronchial epithelial cells during airway inflammation. Am J Respir Cell Mol Biol., 27(2), p. 160-9.

Saxon, A. et al., 1990. Soluble CD23 containing B cell supernatants induce IgE from peripheral blood B-lymphocytes and costimulate with interleukin-4 in induction of IgE. J Allergy Clin Immunol., 86(3 Pt 1), p. 333-44.

Seki, Y. et al., 2003. SOCS-3 regulates onset and maintenance of $\mathrm{T}(\mathrm{H}) 2$-mediated 
allergic responses. Nat Med., 9(8): p. 1047-54.

Singh, A.M. et al., 2007. Bronchiolitis to asthma: a review and call for studies of gene-virus interactions in asthma causation. Am J Respir Crit Care Med., 175(2), p. 108-19.

von Herrath, M.G. and A. Bot., 2003. Immune responsiveness, tolerance and dsRNA: implications for traditional paradigms. Trends Immunol., 24(6), p. 289-93.

Wu, A.H. and W.C. Low., 2003. Molecular cloning and identification of the human interleukin 13 alpha 2 receptor (IL-13Ra2) promoter. Neuro Oncol., 5(3), p. 179-87.

Zhang, Y.et al., 2001. Expression of respiratory syncytial virus-induced chemokine gene networks in lower airway epithelial cells revealed by cDNA microarrays. J Virol., 75(19), p. 9044-58.

Zhou, X. et al., 2007. (Regulation of c-myc expression by Blimp-1 during the differentiation of myeloma cells). Zhonghua Xue Ye Xue Za Zhi., 28(5), p. 318-22. 